\title{
Continuation of Homoclinic Orbits in MATLAB
}

\author{
M. Friedman ${ }^{2}$, W. Govaerts ${ }^{1}$, Yu.A. Kuznetsov ${ }^{3}$, and B. Sautois ${ }^{1}$ \\ 1 Department of Applied Mathematics and Computer Science, Ghent University, \\ Krijgslaan 281-S9, B-9000 Ghent, Belgium \\ 2 Mathematical Sciences Department, University of Alabama, \\ 209 Madison Hall, Huntsville, AL 35899, USA \\ 3 Mathematisch Instituut, \\ Budapestlaan 6, 3584CD Utrecht, The Netherlands
}

\begin{abstract}
We have added the functionality for continuing homoclinic orbits to CL_MATCONT, a user-friendly MATLAB package for the study of dynamical systems and their bifurcations. It is now possible to continue homoclinic-to-hyperbolic-saddle and homoclinic-to-saddle-node orbits. The implementation is done using the continuation of invariant subspaces, with the Ricatti equations included in the defining system. The continuation can be initiated from a limit cycle with large period or from a Bogdanov-Takens point. All known codimension-two bifurcations are tested for, during continuation. The test functions for inclination-flip bifurcations are implemented in a new and more efficient way.
\end{abstract}

\section{Introduction}

A continuous-time dynamical system is usually defined by a set of ordinary differential equations

$$
\dot{x}=f(x, \alpha),
$$

where $x$ is a state vector, $\alpha$ is a parameter vector and $f$ is a smooth function. For general background on dynamical systems theory we refer to the existing literature, in particular [1].

CL_MATCONT [2] and its GUI version MATCONT [3] are MATLAB packages for the study of dynamical systems and their bifurcations. Among other things, they support the numerical continuation of equilibria, limit cycles, limit points, Hopf points, fold, flip, and torus bifurcations of cycles. Both packages are freely available at http://allserv.UGent.be/ ${ }^{\sim}$ ajdhooge. CL_MATCONT and MATCONT are successor packages to AUTO [4] and CONTENT [5], which are written in compiled languages (Fortran, C, C++). The MATLAB platform of CL_MATCONT and MATCONT is attractive because it makes them user-friendly, portable to all operating systems, and allows a standard handling of data files, graphical output, etc. On the other hand, it makes the code inevitably slower because MATLAB is not compiled.

Recently we did some successful testing to improve CL_MATCONT ([6]). First, the transparency and readability of the code was improved greatly by orga- 
nizing it in an object-oriented structure. Eventually, all of CL_MATCONT's total functionality will be transferred into the new structure. Second, a partial inclusion of C-code was used to speed up the computation while preserving the portability of the package. In the case of limit cycle continuations this nearly doubles the speed. This version of CL_MATCONT can be downloaded from http://allserv.UGent.be/ ${ }^{\sim}$ bsautois.

In dynamical systems theory, an orbit corresponding to a solution $\varphi(t)$ is called homoclinic to the equilibrium point $x_{0}$ of (11) if $\varphi(t) \rightarrow x_{0}$ as $t \rightarrow \pm \infty$. There are two types of homoclinic orbits with codimension 1 , namely homoclinicto-hyperbolic-saddle (HHS), if $x_{0}$ is a saddle, and homoclinic-to-saddle-node (HSN), if $x_{0}$ is a saddle-node. Codimension 1 means that in generic dynamical systems with two free parameters these orbits exist along curves in the parameter plane. Both types of homoclinic orbits are important in many applications, e.g. as wave solutions in combustion models 7, to model 'bursting' in models of biological cells [8], chemical reactions [9], etc.

In this paper, we describe new functionalities of CL_MATCONT related to homoclinic orbits. We have implemented continuation of both HHS and HSN orbits, starting from a Bogdanov-Takens (BT) point (no other software allows this) or from a limit cycle with high period, and the detection of a large number of codimension 2 bifurcations during the continuation. To compute the relevant eigenspaces of the equilibrium in each step, we use a method to continue invariant subspaces based on [10. AUTO also has a toolbox for homoclinic continuation, namely HomCont [11. Important differences with our implementation are that HomCont does not use the continuation of invariant subspaces, and cannot start the continuation of homoclinics from a BT-point. Also, we have implemented test functions for inclination flip bifurcations in a new and more efficient way. Thus, the algorithm combines various ingredients from [10, [1], [12] and [13. but differs from any existing implementation.

\section{Extended Defining System for Continuation}

\subsection{Homoclinic-to-Hyperbolic-Saddle Orbits}

To continue HHS orbits in two free parameters, we use an extended defining system that consists of several parts.

First, the infinite time interval is truncated, so that instead of $[-\infty,+\infty]$ we use $[-T,+T]$, which is scaled to $[0,1]$ and divided into mesh-intervals. The mesh is nonuniform and adaptive. Each mesh interval is further subdivided by equidistant fine mesh points. Also, each mesh interval contains a number of collocation points. (This discretization is the same as that in AUTO for boundary value problems.) The equation

$$
\dot{x}(t)-2 T f(x(t), \alpha)=0,
$$

must be satisfied in each collocation point. 
The second part is the equilibrium condition

$$
f\left(x_{0}, \alpha\right)=0 .
$$

Third, there is a so-called phase condition needed for the homoclinic solution, similar to periodic solutions

$$
\int_{0}^{1} \dot{\tilde{x}}^{*}(t)[x(t)-\widetilde{x}(t)] d t=0 .
$$

Here $\widetilde{x}(t)$ is some initial guess for the solution, typically obtained from the previous continuation step. We note that in the literature another phase condition is also used, see, for example [14. However, in the present implementation we employ the condition (4).

Fourth, there are the homoclinic-specific constraints to the solution. For these we need access to the stable and unstable eigenspaces of the system in the equilibrium point after each step. It is not efficient to recompute the spaces from scratch in each continuation-step. Instead, we use the algorithm for continuing invariant subspaces, as described in [10. This method adds two small-sized vectors $\left(Y_{S}\right.$ and $\left.Y_{U}\right)$ to the system variables, from which the necessary eigenspaces (stable and unstable, respectively) can easily be computed in each step.

If $Q_{0}$ is an orthogonal matrix whose first $m$ columns form a basis for the invariant subspace under consideration in the previous step, and $A=f_{x}\left(x_{0}, \alpha\right)$ is the Jacobian at the new equilibrium point, then we first compute the so-called Ricatti-blocks, $T_{i j}$, by the formula

$$
\left[\begin{array}{ll}
T_{11} & T_{12} \\
T_{21} & T_{22}
\end{array}\right]=Q_{0}^{*} A Q_{0} .
$$

If $n$ is the number of state variables, then $T_{11}$ is of size $m \times m$ and $T_{22}$ is $(n-m) \times(n-m)$. This is done for the stable and unstable eigenspaces separately. Now $Y_{S}$ and $Y_{U}$ are obtained from the Ricatti equations

$$
\begin{aligned}
& T_{22 U} Y_{U}-Y_{U} T_{11 U}+T_{21 U}-Y_{U} T_{12 U} Y_{U}=0 \\
& T_{22 S} Y_{S}-Y_{S} T_{11 S}+T_{21 S}-Y_{S} T_{12 S} Y_{S}=0 .
\end{aligned}
$$

Now we can formulate constraints on the behavior of the solution close to the equilibrium $x_{0}$. The initial vector of the orbit, $\left(x(0)-x_{0}\right)$, is placed in the unstable eigenspace of the system in the equilibrium. We express that by the requirement that it is orthogonal to the orthogonal complement of the unstable eigenspace. Using $Y_{U}$, we can compute the orthogonal complement of the unstable eigenspace. If $Q_{0 U}$ is the orthogonal matrix from the previous step, related to the unstable invariant subspace, then a basis for the orthogonal complement in the new step $Q_{1 U_{o}}$ is

$$
Q_{1 U o}=Q_{0 U}\left[\begin{array}{c}
-Y_{U}^{*} \\
I
\end{array}\right] .
$$

Note that $Q_{1 U}$ is not orthogonal. The full orthogonal matrix $Q_{1 U}$ needed for the next step, is computed separately after each step. The equations to be added 
to the system are (after analogous preparatory computations for the stable eigenspace)

$$
\begin{aligned}
& Q_{1 U}^{*}\left(x(0)-x_{0}\right)=0, \\
& Q_{1 S}^{*}\left(x(1)-x_{0}\right)=0 .
\end{aligned}
$$

Finally, the distances between $x(0)$ (resp., $x(1)$ ) and $x_{0}$ must be small enough, so that

$$
\begin{aligned}
& \left\|x(0)-x_{0}\right\|-\epsilon_{0}=0, \\
& \left\|x(1)-x_{0}\right\|-\epsilon_{1}=0 .
\end{aligned}
$$

A system consisting of all equations (2), (3), (4), (6), (7) and (8), is overdetermined. The basic defining system for the continuation of a HHS orbit in two free parameters consists of (2), (3), (6), (7), and (8) with fixed $\epsilon_{0,1}$, so that the phase condition (4) is not used. The variables in this system are stored in one vector. It contains the values of $x(t)$ in the fine mesh points including $x(0)$ and $x(1)$, the truncation time $T$, two free system parameters, the coordinates of the saddle $x_{0}$, and the elements of the matrices $Y_{S}$ and $Y_{U}$. Alternatively, the phase condition (4) can be added if $T$ is kept fixed but $\epsilon_{0}$ and $\epsilon_{1}$ are allowed to vary. It is also possible to fix $T$ and $\epsilon_{0}$, say, and allow $\epsilon_{1}$ to vary, again with no phase condition. Other combinations are also possible, in particular, when the homotopy method [10] is used to compute a starting homoclinic solution.

\section{$2.2 \quad$ Homoclinic-to-Saddle-Node Orbits}

For a homoclinic orbit to a saddle-node equilibrium, the extended defining system undergoes some small changes. Now $\left(x(0)-x_{0}\right)$ has to be placed in the center-unstable subspace. Analogously, $\left(x(1)-x_{0}\right)$ must be in the center-stable subspace. This again is implemented by requiring that the vector is orthogonal to the orthogonal complement of the corresponding space. So the equations (77) themselves do not really change; the changes happen in the computation of the matrices $Q$. The defining system now has one equation less than in the HHS case $\left(n_{s}+n_{u}<n\right.$, with $n_{s}$ the dimension of the stable, and $n_{u}$ of the unstable eigenspace); the number of equations is restored however, by adding the constraint that the equilibrium must be a saddle-node. For this we use the bordering technique, as described in section 4.2 .1 of [15].

\section{$3 \quad$ Starting Strategies}

At present, continuation of homoclinic orbits in CL_MATCONT can be started in two ways: either from a Bogdanov-Takens (BT) point or from a limit cycle with large period.

When starting from a limit cycle with large period, we first look for a point on the cycle with smallest $\|f(x, \alpha)\|$. This point is taken as a first approximation to $x_{0}$. The mesh points of the limit cycle are kept as mesh points for the homoclinic orbit, except for the mesh interval that contains the current equilibrium approximation. In memory, the stored cycle needs to be rotated, so that the first and last point of the orbit $(x(0)$ and $x(1))$ are stored in the correct locations. To 
start from a Bogdanov-Takens point, we use the method from [16]. It computes a predictor for the homoclinic orbit, using the coefficients of the normal form at the Bogdanov-Takens point. However, it does take some trial-and-error to set all parameters for the continuation.

\section{Bifurcations}

Several codimension-two bifurcations can be detected on HHS and HSN curves. In HSN continuation, only one bifurcation is tested for, namely the non-central homoclinic-to-saddle-node orbit or NCHSN. This orbit forms the transition between HHS and HSN curves: a sharp corner, which normally characterizes HHS orbits, appears in the otherwise smooth HSN orbit. The strategy used for detection is taken from HomCont [1].

In HHS continuation, all bifurcations detected in HomCont, are also detected in our implementation. For this, mostly test functions from [11] are used. We refer to that paper for test functions for the following bifurcations:

- Neutral saddle with resonant eigenvalues

- Double real stable leading eigenvalue

- Double real unstable leading eigenvalue

- Neutral saddle, saddle-focus or bi-focus

- Neutrally-divergent saddle-focus (stable)

- Neutrally-divergent saddle-focus (unstable)

- Three leading eigenvalues (stable)

- Three leading eigenvalues (unstable)

- NCHSN

- Shil'nikov-Hopf

- Bogdanov-Takens point

- Orbit-flip with respect to the stable manifold

- Orbit-flip with respect to the unstable manifold

For inclination-flip bifurcations, we also implemented the test functions from [11, but in a more efficient way. We assume that the eigenvalues of $f_{x}\left(x_{0}, \alpha\right)$ are ordered according to

$$
\operatorname{Re} \mu_{n_{s}} \leq \ldots \leq \operatorname{Re} \mu_{1}<0<\operatorname{Re} \lambda_{1} \leq \ldots \leq \operatorname{Re} \lambda_{n_{u}},
$$

where $n_{s}$ is the number of eigenvalues with negative real part, and $n_{u}$ the number of eigenvalues with positive real part. We further assume that $\mu_{1}$ and $\lambda_{1}$ are real and $v_{1}^{S}$ and $v_{1}^{U}$ are the eigenvectors of $f_{x}\left(x_{0}, \alpha\right)$ corresponding to eigenvalues $\mu_{1}$ and $\lambda_{1}$, respectively. Let $y(t)$ satisfy

$$
\begin{aligned}
y^{\prime}(t)+2 T f_{x}^{*}(x(t), \alpha) y(t) & =0, \\
L_{1 S}^{*} y(1) & =0, \\
L_{1 U}^{*} y(0) & =0,
\end{aligned}
$$

where $L_{1 S}$ and $L_{1 U}$ are matrices whose columns form the orthogonal complements to the unstable and stable eigenspaces of $f^{*}\left(x_{0}, \alpha\right)$, respectively. These are defined similar to $Q_{1 S}$ and $Q_{1 U}$ in the previous section. Now introduce 
- a test function for the inclination-flip with respect to the stable manifold:

$$
\psi_{1}=e^{-\mu_{1} T}\left\langle v_{1}^{S}, y(0)\right\rangle=e^{-\mu_{1} T}\left\langle v_{1}^{S}, L_{1 U} \zeta_{2}\right\rangle ;
$$

- a test function for the inclination-flip with respect to the unstable manifold:

$$
\psi_{2}=-e^{-\lambda_{1} T}\left\langle v_{1}^{U}, y(1)\right\rangle=-e^{-\lambda_{1} T}\left\langle v_{1}^{U}, L_{1 U} \zeta_{1}\right\rangle,
$$

where $\zeta_{1}$ and $\zeta_{2}$ are found by demanding that

$$
\left[\begin{array}{lll}
y(1) & \zeta_{1} & \zeta_{2}
\end{array}\right]^{*} \perp \text { Range }\left[\left(D-2 T f_{x}\right)^{*}\left(L_{1 S}^{*} \delta_{1}\right)^{*}\left(L_{1 U}^{*} \delta_{0}\right)^{*}\right]^{*} .
$$

Here $D$ and $\delta$ are the differentiation and the evaluation operators, respectively. Our algorithm keeps track of the left and right singular vectors of this matrix; in each newly computed homoclinic orbit the matrix is bordered with the right and left singular vectors of the previously computed homoclinic orbit to compute the left and right singular vectors of the new matrix.

\section{Example}

The Morris-Lecar model for the barnacle giant muscle fiber 17 is a famous model in computational neuroscience. The equations are:

$C \dot{V}=I_{e x t}-g_{L}\left(V-V_{L}\right)-g_{C a} M_{\infty}\left(V-V_{C a}\right)-g_{K} N\left(V-V_{K}\right), \quad \dot{N}=\tau_{N}\left(N_{\infty}-N\right)$,

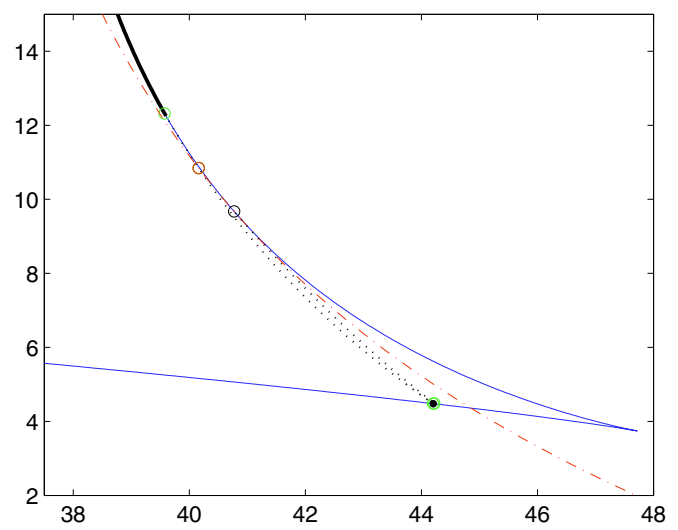

Fig. 1. Bifurcation diagram of the biologically most relevant parameter-range of the Morris-Lecar system. The $\mathrm{X}$-axis shows the value of $I$, the $\mathrm{Y}$-axis that of $V_{3}$. The thin full line is the limit point curve (which clearly shows a cusp point). The dash-dotted line is the Hopf curve. The thick full lines are HSN curves, and the dotted lines are HHS curves. The top and bottom circles indicate the locations of NCHSN points; the bottom circle is actually two circles close together. The second circle from the top is a HNS point, and the third is a BT point 

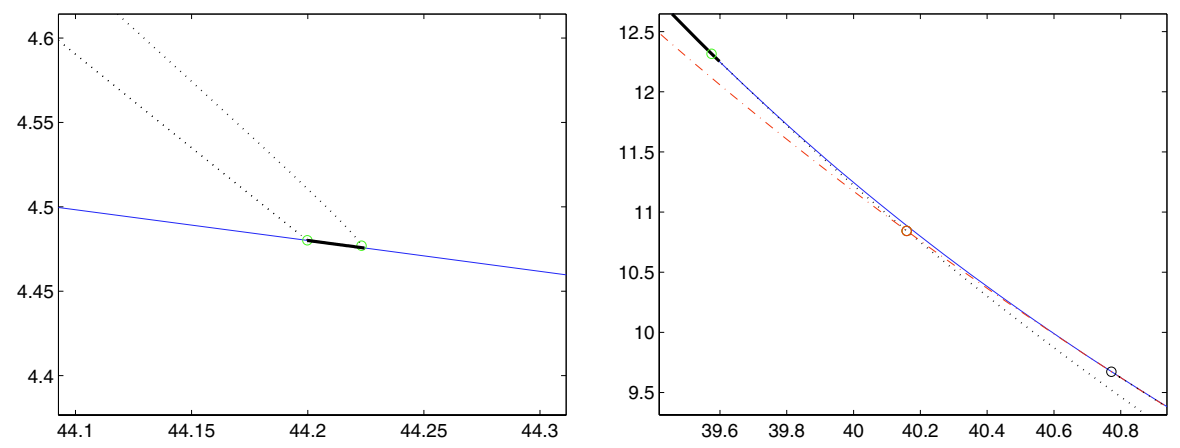

Fig. 2. More detailed views of Figure 1 Left: 2 HHS curves approach the limit point curve. At the intersections, there are NCHSN orbits, and between these, there exists a curve of HSN orbits. Right: Top circle: a NCHSN point forms the transition between HHS and HSN curves. Middle circle: at the intersection of a Hopf curve and a HHS curve (dotted line), a HNS orbit appears. Bottom circle: a BT point exists where a limit point curve (thin solid line) and a Hopf curve (dash-dotted line) touch. From the BT point, a HHS curve arises (which is barely visible here)

where $M_{\infty}=\frac{1}{2}\left(1+\tanh \left(\left(V-V_{1}\right) / V_{2}\right)\right), N_{\infty}=\frac{1}{2}\left(1+\tanh \left(\left(V-V_{3}\right) / V_{4}\right)\right)$ and $\tau_{N}=\phi \cosh \left(\left(V-V_{3}\right) / 2 V_{4}\right)$.

In our tests, $C=5, g_{L}=2, V_{L}=-60, g_{C a}=4, V_{C a}=120, g_{K}=8$, $V_{K}=-80, \phi=\frac{1}{15}, V_{1}=-1.2, V_{2}=18$ and $V_{4}=17.4$ are fixed. $I$ and $V_{3}$ are varied. In the biologically most relevant parameter-range for $I$ and $V_{3}$, a lot of curves and bifurcations are really close together, among others curves of HHS and HSN orbits. In Figure 1 a bifurcation diagram is shown, and more detailed views in Figure 2, These diagrams were computed completely using CL_MATCONT with our modifications and extensions. A full bifurcation diagram and a more thorough study of the model can be found in [18].

Acknowledgement. Bart Sautois thanks the Fund for Scientific Research Flanders - FWO for funding the research reported in this paper. Mark Friedman was supported in part under NSF DMS-0209536.

\section{References}

1. Kuznetsov, Yu.A.: Elements of Applied Bifurcation Theory. 3rd edn. SpringerVerlag, New York, NY (2004).

2. Dhooge, A., Govaerts, W., Kuznetsov, Yu.A., Mestrom, W., Riet, A.M.: A Continuation Toolbox in MATLAB. Manual (2003), http://allserv.UGent.be/ ajdhooge/doc_cl_matcont.zip.

3. Dhooge, A., Govaerts, W., Kuznetsov, Yu.A.: MATCOnT: A Matlab package for numerical bifurcation analysis of ODEs. ACM TOMS 29,2 (2003) 141-164. 
4. Doedel, E.J., Champneys, A.R., Fairgrieve, T.F., Kuznetsov, Yu.A., Sandstede, B., Wang, X.J.: AUTO97: Continuation and bifurcation software for ordinary differential equations (with HomCont), http://indy.cs.concordia.ca/auto (Concordia University, Montreal, Canada, 1998)

5. Kuznetsov, Yu.A., Levitin, V.V.: CONTENT: Integrated environment for analysis of dynamical systems, version 1.5, http://ftp.cwi.nl/CONTENT (CWI, Amsterdam, 1998).

6. Govaerts, W., Sautois, B.: Bifurcation software in Matlab with applications in neuronal modeling. Comput. Meth. Prog. Bio. 77,2 (2005) 141-153.

7. Beresticky, H., Nirenberg, L.: Traveling fronts in cylinders. Ann. Inst. Henri Poincaré 9,5 (1992) 497-572.

8. Rinzel, J., Ermentrout, B.: Analysis of neural excitability and oscillations. In Koch, C., Segev, I. (eds.): Methods in neuronal modeling: from synapses to networks. MIT Press, Cambridge, MA (1989) 135-169.

9. Gray, P., Scott, S.K.: Chemical oscillations and instabilities: non-linear chemical kinetics. Oxford University Press, Oxford, UK (1990).

10. Demmel, J.W., Dieci, L., Friedman, M.J.: Computing connecting orbits via an improved algorithm for continuing invariant subspaces. SIAM J. Sci. Comput. 22,1 (2001) 81-94.

11. Champneys, A.R., Kuznetsov, Yu.A., Sandstede, B.: A numerical toolbox for homoclinic bifurcation analysis. Int. J. Bifurcation Chaos 6,5 (1996) 867-887.

12. Champneys, A.R., Kuznetsov, Yu.A.: Numerical detection and continuation of codimension-two homoclinic bifurcations. Int. J. Bifurcation Chaos 4 (1994) 785822 .

13. Bindel, D., Demmel, J., Friedman, M.: Continuation of invariant subspaces for large bifurcation problems. In proceedings, SIAM Conference on Applied Linear Algebra, July 2003, http://www.siam.org/meetings/la03/proceedings/.

14. Doedel, E.J. and Friedman, M.J.: Numerical computation of heteroclinic orbits, J. Comp. Appl. Math. 26 (1989) 155-170.

15. Govaerts, W.: Numerical Methods for Bifurcations of Dynamical Equilibria. SIAM, Philadelphia (2000).

16. Beyn, W.-J., Champneys, A., Doedel, E., Govaerts, W., Kuznetsov, Yu.A., Sandstede, B.: Numerical continuation, and computation of normal forms. In Fiedler, B. (ed.): Handbook of Dynamical Systems, Vol.2, Ch.4. Elsevier Science (2002).

17. Morris, C., Lecar, H.: Voltage oscillations in the barnacle giant muscle fiber. Biophys. J. 35 (1981) 193-213.

18. Govaerts, W., Sautois, B.: The onset and extinction of neural spiking: a numerical bifurcation approach. To appear in J. Comput. Neurosci. (2005). 